\title{
Cobertura do exame citopatológico na cidade de Pelotas, Brasil
}

\author{
Juvenal Soares Dias da Costa, ${ }^{1}$ Paula Berenhauser D'Elia, ${ }^{2}$ \\ Patrícia Manzolli ${ }^{2}$ e Mônica Regina Moreira ${ }^{3}$
}

RESUMO Em 1995, o câncer de colo de útero foi a quarta causa de morte mais prevalente para o sexo feminino no estado do Rio Grande do Sul, Brasil. Tendo em vista que não existem estudos disponíveis sobre a cobertura do exame citopatológico na cidade de Pelotas - a principal cidade da região sul do estado do Rio Grande do Sul —, em 1992 realizou-se uma pesquisa transversal, de base populacional, entre mulheres de 20 a 69 anos residentes na zona urbana da cidade de Pelotas. O objetivo do estudo foi verificar a realização do exame citopatológico nos 3 anos anteriores à pesquisa; determinar o índice de cobertura deste exame na cidade de Pelotas; e identificar fatores associados à realização do exame. A classe social foi definida como variável sobredeterminante. Na operacionalização de classe social duas classificações foram utilizadas: a classificação de Bronfman e a classificação preconizada pela Associação Brasileira de Institutos de Pesquisa de Mercado. Das 934 mulheres entrevistadas, 606 (65\%) haviam feito o exame citopatológico nos 3 anos que antecederam a pesquisa. A análise multivariada revelou diferenças quanto à realização do exame citopatológico em relação à classe social, idade e freqüência anual de consultas. Não foram encontradas diferenças em relação à escolaridade, tipo de serviço de saúde utilizado e hospitalizações no ano anterior à pesquisa. O estudo conclui que a cobertura do exame citopatológico para mulheres entre 20 e 69 anos em Pelotas é superior à cobertura encontrada em outras partes do país e do mundo. No entanto, nas classes mais bai$x a s, o$ indice de cobertura ficou entre 52 e 56\%. Estes dados podem contribuir para a elaboração de medidas que visem ao aumento da cobertura para setores específicos.

Em 1980, as neoplasias malignas foram responsáveis por $9,5 \%$ das mortes no Brasil. Entre as mulheres com mais de 15 anos, naquele ano, as neoplasias constituíram a segunda causa de mortalidade proporcional, sendo su-

\footnotetext{
Universidade Federal de Pelotas, Faculdade de Medicina, Departamento de Medicina Social. Correspondência e pedidos de separatas devem ser enviados a este autor no seguinte endereço: Departamento de Medicina Social, Faculdade de Medicina, Avenida Duque de Caxias 250, Fragata, CEP 96030-000, Pelotas, RS, Brasil.

2 Universidade Federal de Pelotas, Faculdade de Medicina, Pelotas, Brasil.

3 Universidade Federal de Pelotas, Departamento de Ginecologia e Obstetrícia, Pelotas, Brasil.
}

peradas apenas pelas doenças cárdiocirculatórias. Nesta mesma faixa etária, o câncer cérvico-uterino correspondeu a quase $10 \%$ dos óbitos $(1,2)$. Em 1990, a média de mortalidade por câncer de colo de útero no Brasil, ajustada por idade, foi em torno de 5,5 por 100000 mulheres; vale ressaltar que o subregistro de mortes é de quase 30\% no país (3).

No estado do Rio Grande do Sul, uma série histórica de mortalidade mostrou que os neoplasmas são a segunda causa de morte para todas as idades. Em 1995, o câncer de colo de útero foi a quarta causa de morte mais prevalente para o sexo feminino, com um coeficiente de 5,6 por 100000 mulheres de todas as idades (4).

Pelotas é a principal cidade da região sul do estado do Rio Grande do Sul, Brasil, e possui 289500 habitantes (dados preliminares do recenseamento geral de 1991, divulgados pelo Instituto Brasileiro de Geografia e Estatística na forma de boletim de informações). A zona sul do estado caracteriza-se pela extensão territorial dos municípios e a economia concentra-se nas atividades agropecuárias. Atualmente, esta é a região mais pobre do Rio Grande do Sul. De acordo com a revisão bibliográfica, não existem estudos disponíveis sobre 
a cobertura do exame citopatológico na cidade de Pelotas.

Tendo em vista que a triagem através do exame citopatológico contribui para a redução tanto da morbidade como da mortalidade do câncer cérvicouterino $(5,6)$, o objetivo do presente estudo foi verificar a realização do exame citopatológico em mulheres de 20 a 69 anos nos 3 anos anteriores à pesquisa e determinar o índice de cobertura deste exame na cidade de Pelotas. O estudo procurou, também, identificar fatores associados à realização do exame.

\section{MATERIAIS E MÉTODOS}

O presente artigo descreve um estudo transversal, de base populacional, realizado com mulheres de 20 a 69 anos, residentes na zona urbana de Pelotas, estado do Rio Grande do Sul, Brasil. Este trabalho foi complementar a um estudo mais amplo, realizado entre março e junho de 1992, cujo objetivo foi verificar a utilização de serviços de saúde pela população adulta da cidade de Pelotas $(7,8)$.

Para avaliar a cobertura do exame citopatológico, o tamanho da amostra foi calculado em 504 participantes, considerando um intervalo de confiança de 95\% (IC95\%) para um poder de $95 \%$. A amostra final foi de 934 mulheres, portanto superior à necessária aos objetivos deste estudo. $\mathrm{O}$ tamanho da amostra, mantidas a razão entre expostos e não-expostos e a prevalência dos não-expostos, seria suficiente para um intervalo de confiança de $90 \%$ e um poder de $80 \%$ (risco relativo de até 1,5 ); ou, ainda, seria suficiente para estabelecer um intervalo de confiança de $95 \%$ e poder de $99 \%$, com risco relativo de 2,0 .

A partir do cálculo do tamanho da amostra para o estudo global (1500 participantes) (7), da média de pessoas por domicílio na cidade de Pelotas $(4,0)$, e da proporção na faixa etária de 20 a 69 anos (44\%) (9), estimou-se que um total de 852 domicílios seriam necessários. Em função dos tamanhos amostrais dos outros estudos, realizados concomitantemente com este pro- jeto, foram sorteados 25 setores censitários na zona urbana da cidade de Pelotas. Estabeleceu-se que em cada setor seriam visitados 36 domicílios, totalizando 900 domicílios.

Os domicílios foram selecionados da seguinte forma: um quarteirão em cada setor censitário foi sorteado. O ponto de partida foi escolhido de maneira aleatória. Os entrevistadores sistematicamente entrevistavam um domicílio e pulavam os três seguintes.

As entrevistas foram feitas a partir de questionários padronizados e précodificados, aplicados por estudantes de medicina previamente treinados. Os entrevistadores participaram de um estudo piloto, cujo objetivo foi complementar o treinamento e testar os instrumentos de pesquisa.

A classe social foi definida como variável sobredeterminante. Na operacionalização de classe social duas classificações foram utilizadas. A primeira, elaborada por Bronfman et al. (10) categoriza os indivíduos a partir da posse de meios de produção, de suas inserções nas atividades produtivas e de sua escolaridade. Desta forma, as mulheres foram classificadas nas categorias burguesia, nova e pequena burguesia, pequena burguesia tradicional, proletariado nãotípico, proletariado e sub-proletariado.

A segunda classificação, preconizada pela Associação Brasileira de Institutos de Pesquisa de Mercado (ABIPEME) (11), se baseia na acumulação de bens materiais e na escolaridade. Esta classificação enquadra as pessoas nas classes A, B, C, D ou E, a partir dos escores alcançados.

Coletou-se, também, informações sobre idade, tipo de serviço de saúde onde o exame citopatológico foi realizado, freqüência anual de consultas médicas em geral e hospitalizações no último ano. A variável dependente do estudo referia-se à realização de exame citopatológico nos três anos que antecederam o estudo.

Foram consideradas atualizadas as mulheres que haviam realizado o exame nos 3 anos antes da pesquisa, em função do achado de Day (12), que demonstrou que o risco cumulativo para desenvolver câncer cérvico- uterino invasivo nas idades entre 20 e 64 anos em países ocidentais foi reduzido em $84 \%$ entre mulheres rastreadas a cada 5 anos e em $91 \%$ para mulheres examinadas a cada 3 anos. As mulheres submetidas anualmente ao procedimento teriam uma proteção extra adicional de $2 \%$ (12).

Os questionários foram codificados e, posteriormente, submetidos a duas revisões. A entrada e a limpeza dos dados foi feita através do programa Epi Info.

Os dados foram analisados de forma bivariada e multivariada, com os programas SSPS, Epi Info e Egret. A análise multivariada foi realizada através da regressão logística no programa Egret. Desta forma, as variáveis de classe social foram tratadas para análise como variáveis categóricas ou simuladas (dummies). Na análise multivariada, a classificação de classe social utilizada foi a preconizada pela ABIPEME. A variável de idade também foi tratada como variável categórica (cinco categorias).

\section{RESULTADOS}

Das 934 mulheres entrevistadas, 606 (65\%) haviam feito o exame citopatológico nos 3 anos que precederam o estudo. A tabela 1 mostra as características sócio-demográficas e de utilização de serviços de saúde das mulheres incluídas no estudo.

Como a quantidade de pessoas na categoria burguesia, conforme a classificação de Bronfman et al. (10), era muito pequena (três pessoas) os seus casos foram agrupados, para fins estatísticos, com nova e pequena burguesia. Assim, a categoria nova e pequena burguesia passou a englobar $10 \%$ da população do estudo. Trinta e nove mulheres $(4 \%)$ não se enquadraram em nenhuma categoria da classificação de Bronfman e foram descartadas na análise.

A categorização feita a partir da classificação da ABIPEME também foi transformada para fins estatísticos (apenas oito mulheres se enquadravam na classe $A$ ), mas com liberdade por tratar-se de uma variável quantita- 
TABELA 1. Características das mulheres em relação às variáveis demográficas, socioeconômicas e de utilização de serviços de saúde, Pelotas, Brasil, 1992

\begin{tabular}{|c|c|c|}
\hline Variável & $n=934$ & $\%$ \\
\hline \multicolumn{3}{|l|}{ Classe social (ABIPEMEa) } \\
\hline$A+B$ & 93 & 10 \\
\hline C & 213 & 23 \\
\hline D & 343 & 37 \\
\hline $\mathrm{E}$ & 285 & 30 \\
\hline \multicolumn{3}{|l|}{ Classe social (Bronfman)b } \\
\hline Burguesia/Nova e pequena burguesia & 97 & 11 \\
\hline Pequena burguesia tradicional & 186 & 21 \\
\hline Proletariado não-típico & 414 & 46 \\
\hline Proletariado & 119 & 13 \\
\hline Sub-proletariado & 79 & 9 \\
\hline \multicolumn{3}{|l|}{ Idade } \\
\hline $20-29$ & 228 & 24 \\
\hline $30-39$ & 271 & 29 \\
\hline $40-49$ & 179 & 19 \\
\hline $50-59$ & 152 & 16 \\
\hline $60-69$ & 104 & 11 \\
\hline \multicolumn{3}{|l|}{ Hospitalização no último ano } \\
\hline Não & 835 & 89 \\
\hline Sim & 99 & 11 \\
\hline \multicolumn{3}{|l|}{ Consultas no ano anterior à pesquisa } \\
\hline 7 ou mais & 166 & 18 \\
\hline $6-4$ & 161 & 17 \\
\hline $3-1$ & 404 & 43 \\
\hline Nenhuma & 203 & 22 \\
\hline \multicolumn{3}{|l|}{ Local de consulta } \\
\hline Privado & 148 & 16 \\
\hline Convênio & 139 & 15 \\
\hline Filantrópico & 167 & 18 \\
\hline Público & 242 & 26 \\
\hline Não consultaram & 238 & 25 \\
\hline
\end{tabular}

a Associação Brasileira de Institutos de Pesquisa de Mercado.

b Das 934 mulheres que participaram do estudo, 39 não se enquadraram em nenhuma das categorias da classificação de Bronfman. tiva com pontos de corte arbitrários. Assim, as classes A e B foram agrupadas, também totalizando $10 \%$.

Os resultados da análise bivariada foram descritos nas tabelas 2 e 3 . Os resultados negativos (aqueles cujos IC englobavam o valor unitário ou cujos testes estatísticos estavam acima de $0,05)$ foram mostrados por tratar-se de estudo de base populacional e para servir de comparação com outras pesquisas.

Utilizando-se a classe A e B da classificação da ABIPEME como categoria de base, diferenças significativas foram encontradas em termos da realização do exame citopatológico nos 3 anos que precederam o estudo em relação à classe E. Estas diferenças foram confirmadas pelo risco relativo e respectivos intervalos de confiança.

Conforme a classificação de Bronfman para classe social, não foram encontradas diferenças entre a categoria de base (burguesia e nova e pequena burguesia) e as demais categorias; a comparação entre a categoria de base e a categoria subproletariado ficou muito próxima de um nível significativo $(P=0,06)$ e o limite inferior do IC alcançou um valor maior do que a unidade.

Para analisar a idade, estabeleceu-se o grupo de 20 a 29 anos como categoria de base. Não foram encontradas diferenças entre o grupo de base e o grupo de 40 a 49 anos. Porém, constatou-se que as mulheres entre 30 e 39 anos se submetem mais freqüentemente ao exame citopatológico (tabela 2). Por outro lado, verificou-se (através dos riscos relativos, dos IC e dos testes de associação) que, a partir dos 50 anos, quanto maior a idade, menor era a probabilidade de que as mulheres tivessem se submetido ao exame citopatológico.

Em relação à freqüência anual de consultas médicas em geral, considerou-se como não expostas as mulheres que haviam feito sete ou mais consultas. Nenhuma diferença foi constatada em relação à realização do exame citopatológico nos 3 anos que precederam o estudo entre este grupo e o grupo de mulheres que haviam realizado de quatro a seis consultas durante o ano. As mulheres que não haviam consultado durante o ano, quando comparadas com as da categoria de base (sete ou mais consultas), apresentavam um risco cerca de 1,5 vezes maior para não realização do exame nos 3 anos que precederam a pesquisa.

Em relação ao local de consulta, tendo como categoria de base consultas pelo sistema privado de saúde, não foram encontradas diferenças estatisticamente significativas em relação aos outros sistemas. Mesmo assim, vale ressaltar que a comparação entre o grupo de base e serviços credenciados e conveniados (ou seja, serviços ambulatoriais que atendem sem cobrar diretamente do público, recebendo recursos mediante pagamento através de descontos nos salários ou por valor estipulado pelo governo federal) foi quase estatisticamente significativa $(P=0,08)$.

$\mathrm{Na}$ análise bivariada, em relação à hospitalização no ano que precedeu a pesquisa, verificou-se (através da medida de efeito, de sua precisão e dos testes de associação) que as mulheres hospitalizadas se submetiam mais ao exame citopatológico.

A tabela 4 mostra os resultados da análise multivariada através da regressão logística. Em relação à classe social (ABIPEME), persistiram as diferenças entre a categoria de base (classe A e B) e a classe E. Da mesma forma, perma- 
TABELA 2. Proporção de mulheres que não realizaram o exame citopatológico nos 3 anos que precederam 0 estudo em relação às variáveis demográficas e socioeconômicas, Pelotas, Brasil, 1992

\begin{tabular}{lcccc}
\hline & $\begin{array}{c}\text { Total de } \\
\text { mulheres }\end{array}$ & $\begin{array}{c}\text { Não fizeram exame } \\
n(\%)\end{array}$ & Odds ratio & $\begin{array}{c}\text { Intervalo de confiança } \\
\text { de } 95 \%\end{array}$ \\
\hline Classe social (ABIPEMEa) & & & & \\
Classe A + B & 93 & $25(27)$ & & \\
Classe C & 213 & $59(28)$ & 1,04 & $(0,58$ a 1,87) \\
Classe D & 343 & $119(35)$ & 1,44 & $(0,84$ a 2,49) \\
Classe E & 285 & $125(44)$ & 2,13 & $(1,23$ a 3,68) \\
Classe social (Bronfman) & & & & \\
Burguesia/Nova e pequena & & & & \\
burguesia & 97 & $32(33)$ & - & \\
Pequena burguesia tradicional & 186 & $56(30)$ & 0,88 & $(0,50$ a 1,53) \\
Proletariado não-típico & 414 & $145(35)$ & 1,06 & $(0,67$ a 1,80) \\
Proletariado & 119 & $40(34)$ & 1,03 & $(0,56$ a 1,89) \\
Sub-proletariado & 79 & $38(48)$ & 1,88 & $(0,98$ a 3,64) \\
Idade (anos) & & & & \\
20-29 & 228 & $76(33)$ & - & \\
30-39 & 271 & $65(24)$ & 0,63 & $(0,42$ a 0,95) \\
40-49 & 179 & $57(32)$ & 0,93 & $(0,60$ a 1,45) \\
50-59 & 152 & $66(43)$ & 1,53 & $(0,98$ a 2,40) \\
60-69 & 104 & $64(61)$ & 3,2 & $(1,92$ a 5,33) \\
\hline
\end{tabular}

a Associação Brasileira de Institutos de Pesquisa de Mercado.

b Das 934 mulheres que participaram do estudo, 39 não se enquadraram em nenhuma das categorias da classificação de Bronfman.

TABELA 3. Proporção de mulheres que não realizaram o exame citopatológico nos 3 anos que precederam o estudo em relação às variáveis de utilização de serviços de saúde, Pelotas, Brasil, 1992

\begin{tabular}{lcccc}
\hline & $\begin{array}{c}\text { Total de } \\
\text { mulheres }\end{array}$ & $\begin{array}{r}\text { Não fizeram exame } \\
n(\%)\end{array}$ & Odds ratio & $\begin{array}{c}\text { Intervalo de confiança } \\
\text { de } 95 \%\end{array}$ \\
\hline $\begin{array}{l}\text { Hospitalização no último ano } \\
\text { Não }\end{array}$ & 835 & $304(36)$ & - & \\
Sim & 99 & $24(24)$ & 0,56 & $(0,34$ a 0,93) \\
Consultas no último ano & & & & \\
7 ou mais & 166 & $41(25)$ & - & \\
6-4 & 161 & $29(18)$ & 0,67 & $(0,38$ a 1,18) \\
3-1 & 404 & $138(34)$ & 1,58 & $(1,03$ a 2,43) \\
Nenhuma & 203 & $120(59)$ & 4,41 & $(2,74$ a 7,10$)$ \\
Local de consulta & & & & \\
Privado & 148 & $21(14)$ & - & \\
Conveniado & 139 & $10(7)$ & 0,42 & $(0,17$ a 1,01) \\
Filantrópico & 166 & $27(16)$ & 1,17 & $(0,61$ a 2,27) \\
Público & 241 & $43(18)$ & 1,31 & $(0,72$ a 2,40) \\
Não consultaram & 238 & $228(96)$ & 160,69 & $(69,53$ a 382,62$)$ \\
\hline
\end{tabular}

neceram as diferenças encontradas na análise bivariada por idade (tabela 2).

Em relação à freqüência anual de consultas, a categoria de quatro a seis consultas era a que incluía o maior número de mulheres em dia com o exame citopatológico. A categoria nenhuma consulta evidentemente incluía o maior número de mulheres que não o sistema privado. Entre as mulheres que haviam sido hospitalizadas no ano anterior à pesquisa, quando controladas para classe social e idade, não foram encontradas diferenças significativas em relação à realização de exame citopatológico.

\section{DISCUSSÃO}

A cobertura de exame citopatológico encontrada pelo presente estudo foi de $65 \%$. Esta cobertura superou a preconizada pelo Ministério da Saúde do Brasil no começo da década de 90 $(60 \%)(13)$, embora saiba-se que a efetividade dos programas de controle para o câncer de colo uterino depende de atingir coberturas acima de $80 \%$ (14) e $85 \%$ (15).

Na literatura, para fins de comparação, verificamos, com surpresa, que o nível de cobertura constatado em Pelotas é bastante elevado. Em uma comunidade localizada na periferia da cidade de Pelotas, a cobertura encontrada foi de $21 \%$ (16). Encontraram-se estimativas de cobertura tão baixas como $2 \%$ para o estado do Rio Grande do Sul (1), 7\% para o Brasil (17), 53\% para o estado de São Paulo (18) e 40\% para o Canadá (19). Estes índices devem ser comparados com cautela. A diferença poderia ser justificada pelo critério de se considerar, neste estudo, exame atualizado quando realizado nos 3 anos que precederam a pesquisa. Em outro estudo transversal, realizado na cidade de São Paulo, entre mulheres de 15 a 59 anos, encontrou-se uma cobertura de exame citopatológico de $60,8 \%$ nos três anos que precederam o estudo (20). Uma explicação provável para a alta cobertura encontrada pelo presente estudo é a grande oferta de serviços ambulatoriais na cidade de Pelotas. De qualquer forma, uma restrição do presente estudo foi a impossibilidade de validar os dados, pelas dificuldades amplamente estabelecidas na bibliografia $(21,22)$.

Alguns autores (23-26) associam elevação na incidência de câncer com baixa renda ou baixo nível socioeconômico. Além disso, a classe social parece ter também uma forte correlação com 
TABELA 4. Proporção de mulheres que não realizaram o exame citopatológico nos 3 anos que precederam o estudo em relação à classe social, idade e freqüência anual de consulta, Pelotas, Brasil, 1992

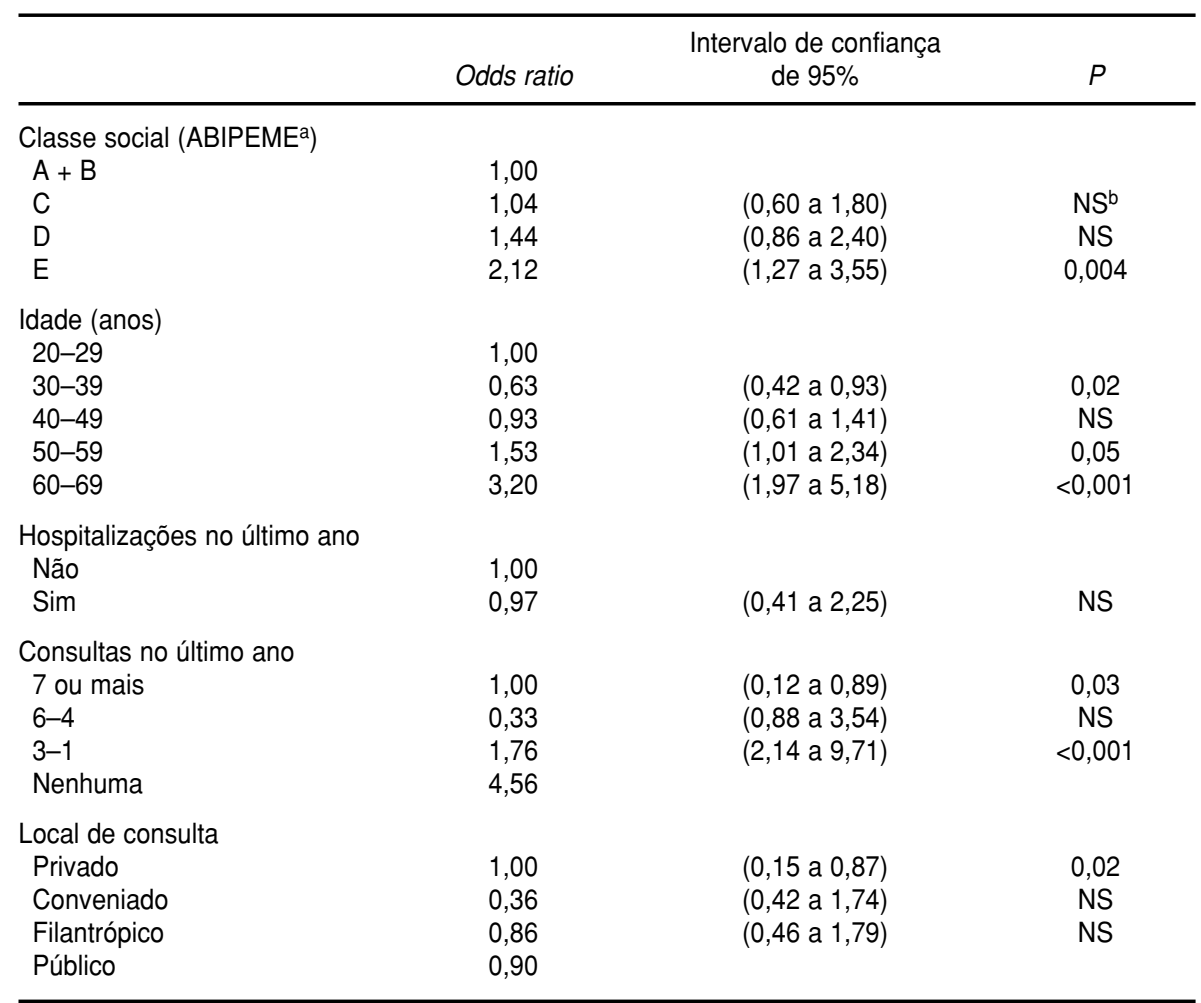

a Associação Brasileira de Institutos de Pesquisa de Mercado.

b Não significativo.

hábitos reprodutivos e sexuais (27). Deve-se ressaltar que esta diferença não ocorreu em relação ao consumo de consultas médicas. Em Pelotas, como possível conseqüência da oferta de serviços de saúde, não se encontrou diferenças entre as classes sociais e a freqüência anual de consultas (8), embora o mesmo estudo tenha demonstrado que existiam diferenças substanciais quanto aos tipos de serviços utilizados em relação à inserção de classe social. $\mathrm{O}$ presente estudo revelou que, quanto mais baixa a classe social, maior o número de mulheres que não realizaram o exame nos 3 anos antes da pes- quisa. Este dado serve para reafirmar a lei dos cuidados inversos (28), ou seja, as pessoas que mais necessitam são as menos assistidas pelo sistema de saúde.

Ainda em relação ao número anual de consultas médicas, seria esperado que as mulheres com mais de sete visitas se submetessem mais ao exame citopatológico. Constatou-se que este grupo de mulheres não estava mais atualizado do que o grupo que consultou de uma a três vezes no ano. Este achado serve como alerta contra o desperdício de oportunidades de prevenção; independentemente da queixa do paciente, sempre que possível deve-se aumentar o espectro da atenção e, com base em critérios epidemiológicos, executar os procedimentos preventivos pertinentes.

A revisão bibliográfica revelou que a incidência de câncer de colo uterino parece aumentar com a idade (29-31). Em nosso estudo, observamos que as mulheres acima de 50 anos são menos examinadas em relação às de 20 a 29 anos. Por outro lado, verificamos que as mulheres de 30 a 39 anos fazem o exame com mais freqüência. Achados semelhantes foram encontrados em outro estudo (32). Uma das razões para este fato seria que, com o decorrer da idade, as consultas médicas em relação a problemas envolvendo a sexualidade e o planejamento familiar diminuem. Outra explicação para este fato poderia ser o efeito de geração, a partir do qual determinadas faixas etárias estariam mais sugestionadas a realizar o exame citopatológico do que outras (32).

Constatou-se que o fato das mulheres passarem por uma internação hospitalar não influenciou a atualização do exame. Este achado aponta para a falta de integralidade nos cuidados médicos: como as mulheres internadas estavam assistidas de forma mais intensiva e em estruturas com maior complexidade, deveriam apresentar cobertura de realização do exame mais elevada.

Em resumo, a partir do presente estudo, concluímos que a cobertura do exame citopatológico para mulheres entre 20 e 69 anos em Pelotas (65\%) é superior à cobertura encontrada em outras partes do país e do mundo. Este índice fala a favor da organização dos serviços de saúde; no entanto, nas classes mais baixas, o índice de cobertura cai de $65 \%$ para $56 \%$ (classificação da ABIPEME) e $52 \%$ (Bronfman). Estes dados podem contribuir para a elaboração de medidas que visem ao aumento da cobertura para setores específicos.

\section{REFERÊNCIAS}

1. Aquino EML, Carvalho AI, Faerstein E, Ribeiro DCS. A situação atual da detecção precoce do câncer cérvico-uterino no Brasil. Cad Saude Publica 1986;2:53-65.
2. Fundação Oswaldo Cruz. Câncer: mortalidade no Brasil. Dados 1991;15:4.

3. Robles SC, White F, Peruga A. Tendencias de la mortalidad por cáncer de cuello de útero en las Américas. Bol Oficina Sanit Panam 1996; 121:478-490.

4. Rio Grande do Sul, Secretaria da Saúde e do Meio Ambiente, Núcleo de Informação em 
Saúde. Estatísticas de saúde: mortalidade 1995. Porto Alegre; Secretaria da Saúde e do Meio Ambiente; 1996

5. Miller AB. Evaluation of the impact of screening for cancer of the cervix. Em: Hakama M, Miller AB, Day NE, eds. Screening for cancer of the uterine cervix. Lyon: International Agency for Research on Cancer; 1986:149-160. (IARC Scientific Publications no 76).

6. Hakama M, Chamberlain J, Day EN, Miller $\mathrm{AB}$, Prorok PC. Evaluation of screening programmes for gynaecological cancer. $\mathrm{Br} J \mathrm{Can}-$ cer 1985;52:669-673.

7. Dias da Costa JS [dissertação de mestrado]. Utilização de serviços ambulatoriais em Pelotas. Pelotas: Faculdade de Medicina da Universidade Federal de Pelotas; 1993.

8. Dias da Costa JS, Fachini LA. Utilização de serviços ambulatoriais em Pelotas: onde a população consulta e com que freqüência. Rev Saude Publica 1997;31:360-369.

9. Fundação Instituto Brasileiro de Geografia e Estatística. Censo demográfico, dados distritais, Rio Grande do Sul, IX Recenseamento Geral do Brasil. Vol 1, tomo 3, no 20. Brasília: IBGE; 1980.

10. Bronfman M, Lombardi C, Facchini LA, Victora CG, Barros FC, Béria JU, et al. Operacionalização do conceito de classe social em estudos epidemiológicos. Rev Saude Publica 1988;22:253-265.

11. Rutter M. Pesquisa de mercado. São Paulo: Editora Ática; 1988.

12. Day NE. The epidemiological basis for evaluation of different screening policies. Em: Hakama M, Miller AB, Day NE, eds. Screening for cancer of the uterine cervix. Lyon: International Agency for Research on Cancer; 1986: 149-160. (IARC Scientific Publications no 76).

13. Brasil, Ministério da Saúde. Plano qüinqüenal de saúde 1990-1995. Brasília: Ministério da Saúde; 1990.

14. Organización Panamericana de la Salud, Programa de Salud del Adulto y Programa de
Salud MaternoInfantil, con la colaboración de Paola G, González J, Ruiz PA, Sepúlveda C. Guías para la evaluación de los programas de detección precoz del cáncer de cuello uterino. Bol Oficina Sanit Panam 1989;107:454-457.

15. Brasil, Ministério da Saúde. Controle do câncer cérvico-uterino e de mama: normas e manuais técnicos. Rio de Janeiro: Ministério da Saúde/INCA; 1994.

16. Fassa AG [dissertação]. Relato da experiência de trabalho com a comunidade do Sítio Floresta enfatizando a participação popular. Santa Maria: Curso de Pós-Graduação em Saúde Coletiva da Universidade Federal de Santa Maria; 1990.

17. Brasil, Ministério da Saúde, Instituto Nacional do Câncer. Câncer do colo do útero: tema do Dia Nacional de Combate ao Câncer. Prev \& Cancer 1994;I:1.

18. Pinotti JA, Faúndes A, Hardy EE, Simões IR, Osis MJD, Souza TR, Moraes TM. Avaliação da assistência ginecológica no estado de São Paulo. Rev Ginecol Obstet 1990;1:7-21.

19. Anderson GH, Boyes DA, Benedet JL, Le Riche JC, Matisic JP, Suen KC, Worth AJ, Millner A. Organization and results of the cervical cytology screening programme in British Columbia, 1955-85. Br Med J 1988;296:975-978.

20. Nascimento CMR, Eluf-Neto J, Rego RA. Cobertura do teste de Papanicolaou no município de São Paulo e características das mulheres que realizaram o teste. Bol Oficina Sanit Panam 1996;121:491-501.

21. Kroeger A. Health interview surveys in developing countries: review of the methods and results. Int J Epidemiol 1983;12:465-481.

22. Ross DA, Vaughan JP. Health surveys in developing countries: a methodological review with recommendations for future surveys. London: School of Hygiene and Tropical Medicine; 1984. (EPC Publications no 4).

23. Foltz AM, Kelsey JL. The annual Pap test: a dubious policy success. World Health Forum 1980;I:105-116.
24. Herrero R, Brinton LA, Reeves WC, Brenes MM, Terraro F, Britton R, et al. Factores de riesgo de carcinoma invasor del cuello uterino en América Latina. Bol Oficina Sanit Panam 1990;109:6-23.

25. Brasil, Ministério da Saúde, Instituto Nacional do Câncer. Controle do câncer: uma proposta de integração ensino-serviço. Rio de Janeiro: ProOnco; 1993.

26. Restrepo HE, González J, Roberts E, Litvak J. Epidemiología y control del cáncer del cuello uterino en América Latina y el Caribe. Bol Oficina Sanit Panam 1987;102:578-592.

27. Hakama M. Cervical cancer: risk groups for screening. Em: Hakama M, Miller AB, Day $\mathrm{NE}$, eds. Screening for cancer of the uterine cervix. Lyon: International Agency for Research on Cancer; 1986:213-219. (IARC Scientific Publications no 76).

28. Hart JT. The inverse care law. Lancet 1971; 1:405-412.

29. Bixby LB, Vázquez CG. Epidemiología descriptiva del cáncer de mama y del cuello de útero en Costa Rica. Bol Oficina Sanit Panam 1987;105:483-493.

30. Macgregor JE, Teper S. Mortality from carcinoma of cervix uteri in Britain. Lancet 1978; 2:774-776.

31. Tognini PHF. Carcinoma in situ do colo uterino. J Bras Ginecol 1988;98:325-328.

32. Chamberlain J. Reasons that some screening programs fail to control cervical cancer. Em: Hakama M, Miller AB, Day NE, eds. Screening for cancer of the uterine cervix. Lyon: International Agency for Research on Cancer; 1986: 213-219. (IARC Scientific Publications no 76).

Manuscrito recebido em 23 de junho de 1995. Aceito em versão revisada em 10 de abril de 1997.
SUMMARY

\section{Pap smear test coverage in the city of Pelotas (Rio Grande do Sul), Brazil}

In 1995, cervical cancer was the fourth most important cause of death among women in the state of Rio Grande do Sul, Brazil. There are no available studies on Pap smear examination coverage in Pelotas, which is the largest city in the southern part of Rio Grande do Sul. Accordingly, in 1992 a population-based, cross-sectional study was carried out among 20 to 69 year-old women residing in urban areas of the city. The objectives of the study were to ascertain any Pap smear testing in the 3 years prior to the study, to determine the coverage of cervical cancer screening, and to identify any factors associated with the performance of Pap tests in Pelotas. Social class was chosen as the determining variable. To categorize social class, two classifications were used: Bronfman's classification and the classification used by the Brazilian Association of Market Research Institutes. Among the 934 women interviewed, 606 (65\%) had had a Pap smear test in the 3 years before the study. The multivariate analysis revealed significant differences regarding performance of such tests in terms of social class, age, and frequency of medical consultations per year. No significant differences were found in terms of education, type of health service used, or hospitalizations during the year prior to the study. This study reveals that coverage of cervical cancer screening for women between 20 and 69 years of age in Pelotas is higher than the coverage found in other parts of the country or even of the world. However, for women in lower social classes, coverage was found to be between 52 and $56 \%$. These data can contribute to the development of measures aimed at increasing cervical cancer screening among specific sectors of the population. 